\title{
Comparative study of cisplatin-based definitive concurrent chemoradiotherapy with S-1 versus paclitaxel for unresectable locally advanced esophageal squamous cell carcinoma
}

\author{
Min Fang ${ }^{1, *}$, Tao Song ${ }^{1, *}$, Xiaodong Liang ${ }^{1}$, Shiliang Lv $^{1}$, Jianbo Li $^{2}$, Hong'en $\mathrm{Xu}^{1}$, \\ Limin Luo ${ }^{1}$, Yongshi Jia ${ }^{1}$ \\ ${ }^{1}$ Department of Radiation Oncology, Zhejiang Provincial People's Hospital, Hangzhou 310000, Zhejiang, P. R. China \\ ${ }^{2}$ Department of Radiation Oncology, Ningbo Mingzhou Hospital, Ningbo 315000, Zhejiang, P. R. China \\ "These authors contributed equally to this work \\ Correspondence to: Limin Luo, email: luoliminflk@163.com \\ Yongshi Jia, email: jyssrmyy@163.com
}

Keywords: definitive concourrent chemoradiotherapy, esophageal squamous cell carcinoma, S-1, cisplatin, paclitaxel

Received: June 20, $2016 \quad$ Accepted: March 03, $2017 \quad$ Published: March 14, 2017

Copyright: Fang et al. This is an open-access article distributed under the terms of the Creative Commons Attribution License (CC-BY), which permits unrestricted use, distribution, and reproduction in any medium, provided the original author and source are credited.

\section{ABSTRACT}

This study compared the efficiency and safety of definitive concurrent chemoradiotherapy (CCRT) using Paclitaxel plus Cisplatin (TP) versus S-1 plus Cisplatin (CS) in unresectable locally advanced esophageal squamous cell carcinoma (LAESCC). Between January 2009 and December 2013, 203 LAESCC patients were retrospectively reviewed. We performed a propensity score matching analysis; 41 patients treated with the CS regimen were matched 1:1 to patients who received the TP regimen. Patient- and disease-related characteristics were well-balanced between the two groups. The CS group showed significantly better treatment compliance $(90.2 \%$ vs. $70.7 \%, P=0.026)$ and less hospital stay (48 days vs 49 days, $P=0.025$ ) over the TP group during the CCRT course. The complete response rate was comparable between the two groups (51.2\% vs. $48.8 \%$, $P=0.825$ ). The 1- and 3-year overall survival (OS) rates in the TP group were $63.4 \%$ and $32.4 \%$ compared to $62.8 \%$ and $32.1 \%$ in the CS group, respectively $(P=0.796)$. The $1-$ and 3-year progression-free survival (PFS) rates in the TP group were $51.2 \%$ and $24.9 \%$, compared to $53.6 \%$ and $18.9 \%$ in the CS group, respectively $(P=0.630)$. The incidence of severe and total neutropenia in the TP group was significantly higher compared to the CS group ( $P=0.011$ and 0.046 , respectively). Multivariate analysis revealed that $\mathrm{T}$ stage and the complete response rate were strong prognostic factors associated with $0 S$ and PFS. In conclusion, both treatment regimens yielded satisfactory survival outcomes, but the CS regimen could significantly improve treatment compliance, reduce hematological toxicities and lengths of hospital stay. Future prospective studies in large cohorts are highly warranted to confirm the findings in our report.

\section{INTRODUCTION}

Patients presenting with carcinoma of the esophagus continue to represent one of the most significant health problems, and its associated morbidity is still increasing worldwide. Despite advances in diagnosis for early-stage cancers, the majority of patients are diagnosed at advanced stages [1-3]. Definitive concurrent chemoradiotherapy (CCRT) is the standard treatment option for unresectable locally advanced diseases. The seminal phase III trial of the Radiation Therapy Oncology Group (RTOG) 85-01 compared the efficacy of CCRT, which consisted of 5-fluorouracil $(5-\mathrm{Fu})$ plus Cisplatin (CDDP) with radiotherapy (RT) alone and resulted in a long-term survival rate of $26 \%$ in the CCRT group. However, the efficacy of this regimen was only approximately $25 \%-35 \%$ while severe and fatal side effects were observed in $44 \%$ and $20 \%$ of the patients [4]. Attempts have been made to further improve the therapeutic ratio and to reduce toxic reactions for esophageal cancer. 
Paclitaxel (PTX), a broad-spectrum cytotoxic drug, has been shown to be a promising treatment agent against esophageal cancer and a series of phase II studies has demonstrated favorable response rates and comparable survival outcomes to $5-\mathrm{Fu} / \mathrm{CDDP} / \mathrm{RT}$ regimens $[5,6]$. RTOG 0113 trial, which compared the efficacy of CCRT with the TP (PTX and CDDP) regimen, showed that median survival time was 14.9 months, and the 1- and 2-year OS rates were $69 \%$ and $37 \%$, respectively. Severe (grade $\geq 3$ ) side effects were observed in greater than $40 \%$ patients [7]. Tang HR et al. reported the median OS and PFS time was 28.5 and 14.7 months, and the 1- and 2 -year survival rates were $75 \%$ and $54 \%$, respectively with grades 3 and 4 neutropenia occurred in $30.3 \%$ and $31.6 \%$ in inoperable ESCC patients who received CCRT with a 3-week schedule of TP regimen [8]. Thus, the current National Comprehensive Cancer Network (NCCN) guidelines for esophageal cancer recommend definitive CCRT based on 5-Fu or Taxane for patients who refused surgery or were medically unfit for esophagectomy. However, adverse effects associated with PTX and Cremophor/ethanol, including acute hypersensitivity and neurotoxicity, were still observed in nearly $40 \%$ patients, which limited its implementation in the clinic [9].

The compound drug, S-1 (TS-1, Taiho Pharmaceutical), consists of a combination of tegafurgimeracil-oteracil. S-1 has been widely used in a variety of solid tumors, including esophageal cancer. Preclinical studies indicated that S-1 demonstrates far superior antitumor activities than 5-Fu and enhances the sensitivity of cancer cells to the effects of RT $[10,11]$. Accumulating clinical evidence also supports the opinion that the combination of CDDP and S-1(CS) represents a viable treatment regimen for esophageal cancer [12, 13]. In studies performed in Japan, the CS regimen combined with RT in the setting of CCRT achieved encouraging response rates of $64.4 \%--89.7 \%$. In addition, toxicities associated with CS are modest, which enabled their concomitant use with RT for esophageal cancer patients $[14,15]$.

Considering the high-grade evidence for the use of the CS regimen combined with RT in locally advanced esophageal squamous cell carcinoma (LAESCC) is in shortage, we performed this retrospective study to compare the clinical outcomes of CS versus TP with RT for unresectable LAESCC, focusing on treatment compliance, response rate, toxicity, and survival outcomes through propensity score matching (PSM) analysis.

\section{RESULTS}

\section{Demographic variables of the enrolled patients}

After PSM analysis, 82 well-balanced unresectable LAESCC patients were available for outcome comparison (41 patients in each group, Figure 1) and the median value of the PSM score for the TP and CS group was 0.423 (range: $0.154-0.840$ ) and 0.422 (range: 0.154-0.814). No significantly demographic difference was observed among these two groups (Table 1).Within the whole series, the median patient age was 58 years (range, 38-77 years). The mean length of the primary tumor was $6.2 \mathrm{~cm}$ (range, $2.0-12.5 \mathrm{~cm}$ ). Nearly half of the tumors were located in the upper-third of the esophagus (46.3\%). Fifty-eight (70.7\%) patients had stage II-III diseases while 24 (29.3\%) patients were diagnosed with stage IVa. Reasons for indication of definitive CCRT for stage II ESCC patients were: cervical esophagus $(n=21)$ and rejection of esophagectomy $(n=4)$.

\section{Treatment compliance and tumor response}

All patients in the TP group completed the first cycle of chemotherapy. Three patients refused the second cycle of chemotherapy for occurring grade 4 leukocytopenia, and these patients also refused radiotherapy. Five (12.2\%) patients required a dose reduction in the second cycle of chemotherapy for hematological toxicities. Thirty-eight patients completed radiotherapy, including 3 patients with radiation delay and 1 patient with fatal esophagitis. Thus, $29(70.7 \%)$ patients completed the preplanned CCRT on schedule.

Among the 41 patients in the CS group, 39 (95.1\%) patients received the full dose of radiotherapy, whereas two patients required radiation delay or dose reduction due to grade 3 or higher esophagitis. Two additional patients required a dose reduction of S-1 for hematological toxicities. Thus, a total of $37(90.2 \%)$ patients completed S-1/CDDP/RT without changing the treatment dose. The difference in treatment compliance during CCRT between the two groups was statistically significant $(P=0.026)$. In addition, lengths of hospital stay and hospitalization expenses during CCRT course were also collected for the two groups. The median length of hospital stay and medical cost for TP group were 49 days (range: 44-61 days) and 12.70 thousand dollars person-times (range: 10.50-17.20 thousand dollars). The corresponding figures for CS group were 48 days (range: 44-57 days) and 12.90 thousand dollars person-times (range: 10.8016.10 thousand dollars). By comparing to the TP group, CS group significantly decreased the length of hospital stay $(P=0.025)$ and the inpatient expenses was not statistically significant $(P=0.904)$. After completing CCRT, 11 (26.8\%) patients in the TP group completed the additional full 4 cycles of chemotherapy while 18 (43.9\%) patients in the CS group received the additional 4 cycles of maintenance chemotherapy. This difference was not statistically significant $(P=0.106)$. The median number of chemotherapy cycles was five for both treatment regimens while the total number of cycles delivered was 186 and 199 for TP and CS groups, respectively.

The tumor response was documented using RECIST. In the TP group, complete response (CR) was observed in 21 patients $(51.2 \%)$, partial response (PR) 
Table 1: Patients' background characteristics

\begin{tabular}{|c|c|c|c|c|}
\hline Characteristic & $\begin{array}{c}\text { Total } \\
(n=82), \%\end{array}$ & $\begin{array}{c}P T X / C D D P / R T \\
(n=41), \%\end{array}$ & $\begin{array}{c}S-1 / C D D P / R T \\
(n=41), \%\end{array}$ & $P$ value \\
\hline Age(years) & & & & 0.823 \\
\hline age $<58$ & $35(42.7)$ & $18(43.9)$ & $17(41.5)$ & \\
\hline age $\geq 58$ & $47(57.3)$ & $23(56.1)$ & $24(58.5)$ & \\
\hline Gender & & & & 0.532 \\
\hline Female & $12(14.6)$ & $5(12.2)$ & $7(17.1)$ & \\
\hline Male & $70(85.4)$ & $36(87.8)$ & $34(82.9)$ & \\
\hline ECOG Performance Status & & & & 1.000 \\
\hline $0-1$ & $58(70.7)$ & $29(70.7)$ & $29(70.7)$ & \\
\hline 2 & $24(29.3)$ & $12(29.3)$ & $12(29.3)$ & \\
\hline T Stage & & & & 0.659 \\
\hline $\mathrm{T}_{3}$ & $40(48.8)$ & $21(51.2)$ & $19(46.3)$ & \\
\hline $\mathrm{T}_{4}$ & $42(51.2)$ & $20(48.8)$ & $22(53.7)$ & \\
\hline N Stage & & & & 0.656 \\
\hline $\mathrm{N}_{0}$ & $46(56.1)$ & $24(58.5)$ & $22(53.7)$ & \\
\hline $\mathrm{N}_{1}$ & $36(43.9)$ & $17(41.5))$ & $19(46.3)$ & \\
\hline M Stage & & & & 0.627 \\
\hline $\mathrm{M}_{0}$ & $58(70.7)$ & $30(73.2)$ & $28(68.3)$ & \\
\hline $\mathrm{M}_{1 \mathrm{a}}$ & $24(29.3)$ & $11(26.8)$ & $13(31.7)$ & \\
\hline Clinical Stage (AJCC 2002) & & & & 0.787 \\
\hline Stage II & $25(30.5)$ & $12(29.3)$ & $13(31.7)$ & \\
\hline Stage III & $33(40.2)$ & $18(43.9)$ & $15(36.6)$ & \\
\hline Stage $\mathrm{IV}_{\mathrm{a}}$ & $24(29.3)$ & $11(26.8)$ & $13(31.7)$ & \\
\hline Tumor Location & & & & 0.811 \\
\hline Upper-third & $38(46.3)$ & $19(46.3)$ & $19(46.3)$ & \\
\hline Middle-third & $30(36.6)$ & $16(39.0)$ & $14(34.1)$ & \\
\hline Lower-third & $14(17.1)$ & $6(14.7)$ & $8(19.6)$ & \\
\hline Histological Differentiation & & & & 0.890 \\
\hline Well differentiated & $19(23.2)$ & $10(24.4)$ & $9(22.0)$ & \\
\hline Fairly differentiated & $37(45.1)$ & $19(46.3)$ & $18(43.9)$ & \\
\hline Poorly differentiated & $26(31.7)$ & $12(29.3)$ & $14(34.1)$ & \\
\hline Tumor Length (cm) & & & & 1.000 \\
\hline$<5$ & $32(39.0)$ & $16(39.0)$ & $16(39.0)$ & \\
\hline$\geq 5$ & $50(61.0)$ & $25(61.0)$ & $25(61.0)$ & \\
\hline Weight Loss in 6 months & & & & 0.810 \\
\hline$\leq 10 \%$ & $57(69.5)$ & $29(70.7)$ & $28(68.3)$ & \\
\hline$>10 \%$ & $25(30.5)$ & $12(29.3)$ & $13(31.7)$ & \\
\hline
\end{tabular}

Abbreviations: n: number of patients; PTX: Paclitaxel: CDDP: Cisplatin; RT: radiotherapy; ECOG: Eastern Cooperative Oncology Group. Upper: including cervical and upper thoracic portion; Middle: Mid-thoracic portion; Lower: including lower thoracic and distal esophagus. 
in 13 patients $(31.7 \%)$, stable disease (SD) in 4 patients $(9.8 \%)$, and progressive disease (PD) in $3(7.3 \%)$ patients, yielding an objective response rate (ORR) of $82.9 \%$. In addition, in the CS group, 34 patients achieved an ORR of $82.9 \%(C R=20$ and $P R=14)$ and 2 patients exhibited PD. No significant difference in the $\mathrm{CR}$ and ORR rates were observed between the two groups $(P=0.825$ and 1.000 , respectively).

\section{Acute and late toxic reactions}

Acute treatment-related toxicity could be evaluated in all cases (Table 2). In general, patients in the TP group suffered from more treatment toxicities than those who received the CS regimen. There was grade 3-4 hematologic toxicity in $48.8 \%$ of patients receiving TP versus $31.7 \%$ of patients who received $\mathrm{CS}(P=0.115)$. TP was associated with a significantly higher rate of severe and fatal neutropenia than CS $(P=0.011)$. The occurrence of severely non-hematologic toxicity was not significantly different between the two groups. A comparison of the total incidence of hematological toxicities revealed that the CS group also had a significant lower incidence of neutropenia over the TP group $(P=0.046)$. For nonhematological toxicity, the TP group demonstrated a significantly higher incidence of neurotoxicity compared with the CS group $(P=0.034)$. During maintenance chemotherapy, the TP regimen had a higher incidence of nausea/vomiting compared with the CS group $(P=0.046)$. Hematological toxicities were comparable in both treatment groups. There were no treatment-related toxic deaths during the treatment course.

Late grade $\geq 3$ esophageal toxicities were observed in four patients in the TP group and 5 patients in the CS group. The incidences of late esophageal, lung and heart toxicities showed no significant difference between the two groups.

\section{Survival and prognostic analysis}

The median follow-up period was 28.4 months. The median OS of the overall population was 21.6 months (95\% CI: 15.4-27.8 months), and the 1-year OS rates were $63.4 \%$ and $62.8 \%$ for TP group and CS group, respectively. The 3 -year OS rates were $32.4 \%$ and $32.1 \%$ for TP and CS group, respectively. The difference in OS was not statistically significant $(P=0.796)$.

During the follow-up period, 31 patients had progressive disease in the TP group. Primary failure sites included the following: 14 locoregional and residual disease, 12 distant, and 5 in both sites. In the CS group, 32 patients experienced treatment failure, and the

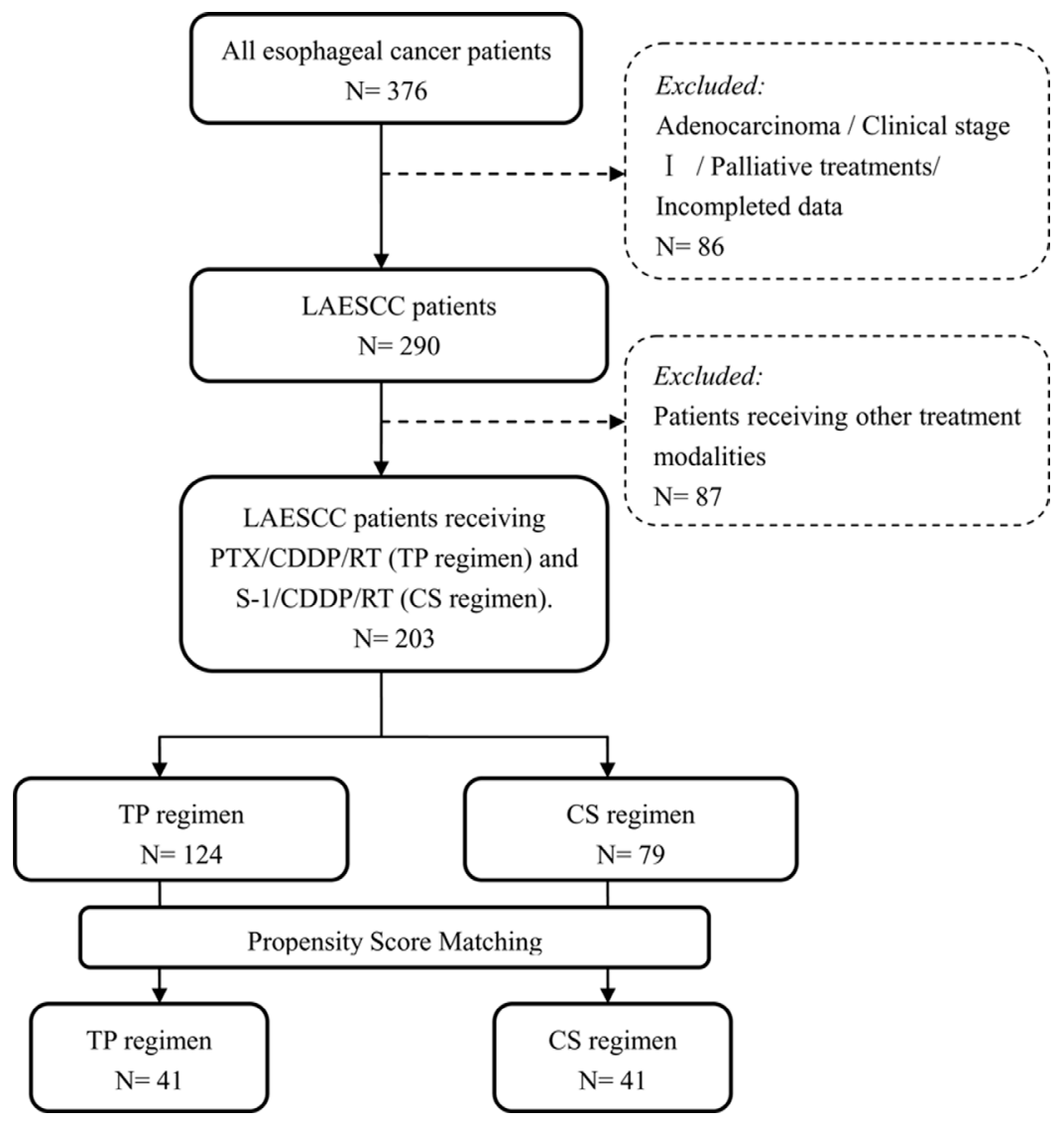

Figure 1: Patient disposition. 
Table 2: Treatment-related toxicities

\begin{tabular}{|c|c|c|c|}
\hline Event & $\begin{array}{c}\text { PTX/CDDP/RT } \\
(n=41), \% \\
\end{array}$ & $\begin{array}{c}\text { S-1/CDDP/RT } \\
(n=41), \% \\
\end{array}$ & $P$ value \\
\hline \multicolumn{4}{|l|}{ Events of grade $\geq 3$ during CCRT } \\
\hline Leucocytopenia & $14(34.1)$ & $10(24.4)$ & 0.332 \\
\hline Neutropenia & $20(48.8)$ & $9(22.0)$ & 0.011 \\
\hline Anemia & $6(14.6)$ & $3(7.3)$ & 0.480 \\
\hline Thrombocytopaenia & $5(12.2)$ & $4(9.8)$ & 1.000 \\
\hline Esophagitis & $4(9.8)$ & $2(4.9)$ & 0.672 \\
\hline Anorexia & $1(2.4)$ & $2(4.9)$ & 1.000 \\
\hline Nausea/Vomiting & $3(7.3)$ & $2(4.9)$ & 1.000 \\
\hline Diarrhea & $2(4.9)$ & $1(2.4)$ & 1.000 \\
\hline Fatigue & $3(7.3)$ & $2(4.9)$ & 1.000 \\
\hline Pneumonitis & $1(2.4)$ & $2(4.9)$ & 1.000 \\
\hline \multicolumn{4}{|l|}{ Events of any grade during CCRT } \\
\hline Leucocytopenia & $35(85.4)$ & $28(68.3)$ & 0.067 \\
\hline Neutropenia & $37(90.2)$ & $30(73.2)$ & 0.046 \\
\hline Anemia & $25(61.0)$ & $27(65.9)$ & 0.647 \\
\hline Thrombocytopaenia & $16(39.0)$ & $14(34.1)$ & 0.647 \\
\hline Esophagitis & $34(82.9)$ & $38(92.7)$ & 0.177 \\
\hline Anorexia & $22(53.7)$ & $28(68.3)$ & 0.174 \\
\hline Nausea/Vomiting & $23(56.1)$ & $17(41.5)$ & 0.185 \\
\hline Diarrhea & $17(41.5)$ & $12(29.3)$ & 0.248 \\
\hline Fatigue & $26(63.4)$ & $22(53.7)$ & 0.370 \\
\hline Pneumonitis & $7(17.1)$ & $7(17.1)$ & 1.000 \\
\hline Liver function & $5(12.2)$ & $8(19.5)$ & 0.364 \\
\hline Nephrotoxicity & $2(4.9)$ & $1(2.4)$ & 1.000 \\
\hline Neurotoxicity & $8(19.5)$ & $1(2.4)$ & 0.034 \\
\hline Constipation & $2(4.9)$ & $4(9.8)$ & 0.672 \\
\hline Mucositis & $3(7.3)$ & $2(4.9)$ & 1.000 \\
\hline Events of any grade during maintenance $\mathrm{CT}$ & $\begin{array}{c}\text { PTX/CDDP/RT } \\
(n=38), \%\end{array}$ & $\begin{array}{c}\text { S-1/CDDP/RT } \\
(n=39), \%\end{array}$ & $P$ value \\
\hline Leucocytopenia & $15(39.5)$ & $9(23.1)$ & 0.120 \\
\hline Neutropenia & $11(28.9)$ & $14(35.9)$ & 0.515 \\
\hline Anemia & $4(10.5)$ & $10(25.6)$ & 0.086 \\
\hline Thrombocytopaenia & $5(13.2)$ & $8(20.5)$ & 0.389 \\
\hline Nausea/Vomiting & $15(39.5)$ & $7(17.9)$ & 0.037 \\
\hline Diarrhea & $6(15.8)$ & $4(10.3)$ & 0.702 \\
\hline Fatigue & $7(18.4)$ & $5(12.8)$ & 0.498 \\
\hline Liver function & $3(7.9)$ & $2(5.1)$ & 0.976 \\
\hline Nephrotoxicity & $5(13.2)$ & $3(7.7)$ & 0.680 \\
\hline \multicolumn{4}{|l|}{ Late grade $\geq 3$ toxicity } \\
\hline Esophagus & $4(9.8)$ & $5(12.2)$ & 1.000 \\
\hline Lung & $2(4.9)$ & $1(2.4)$ & 1.000 \\
\hline Heart & $1(2.4)$ & $2(4.9)$ & 1.000 \\
\hline
\end{tabular}


corresponding figures were 17 (41.5\%), 10 (24.4\%) and 5 cases $(12.2 \%)$. The median PFS was 13.2 months $(95 \%$ CI: 7.4-19.1 months), and the 1- and 3-year PFS rates were $51.2 \%, 24.9 \%$ and $53.6 \%, 18.9 \%$ for the TP group and CS group, respectively. No statistically significant difference was found when comparing group survival times for PFS $(P=0.630$, Figure 2$)$.

Univariate analyses were performed to assess the predictive capability of each variable (Table 3 ). These results suggested that several covariates were significantly associated with OS: ECOG PS (HR 2.416, $P=0.002$ ), T stage (HR 4.101, $P=0.000$ ), $\mathrm{N}$ stage (HR 2.387, $P=0.002)$, M stage (HR 2.129, $P=0.010)$, clinical stage (HR 1.892, $P=0.000$ ), and clinical response (HR 3.898, $P=0.000)$. The variables significantly associated with PFS were: ECOG PS (HR 1.976, $P=0.013$ ), T stage (HR $2.570, P=0.000)$, N stage (HR 2.304, $P=0.002)$, clinical stage (HR 1.537, $P=0.009)$, and clinical response (HR $3.057, P=0.000)$.

To identify independent prognostic factors, the factors that were found to be significant using univariate analysis were subjected to multivariate analysis. Multivariate analysis revealed that T stage $(P=0.009$ and 0.042 , respectively), $\mathrm{N}$ stage $(P=0.027$ and 0.014 , respectively) and clinical response $(P=0.002$ and 0.012 , respectively) were independent factors affecting OS and PFS (Table 4).

Furthermore, the 1- and 3-year OS rates and the median OS time for 124 patients with TP regimen were $76.9 \%, 36.1 \%$, and 24.9 months, respectively, and $71.6 \%$, $30.6 \%, 23.1$ months, respectively, for all patients received CS regimen. The 1- and 3-year PFS rates were $71.4 \%$, $26.3 \%$ and $66.8 \%, 23.8 \%$ for the TP group and CS group, respectively. No significant difference was found for both OS and PFS in two groups ( $P=0.660$ and 0.684 , respectively). Multivariate analysis among all patients revealed that $\mathrm{T}$ stage (OS: HR 1.846, $P=0.002$; PFS: HR 1.758, $P=0.002$ ) and clinical response (OS: HR 2.783, $P=0.000$; PFS: HR 2.207, $P=0.000)$ were strong prognostic factors associated with OS and PFS. In addition, M stage (HR 2.482, $P=0.016)$ and

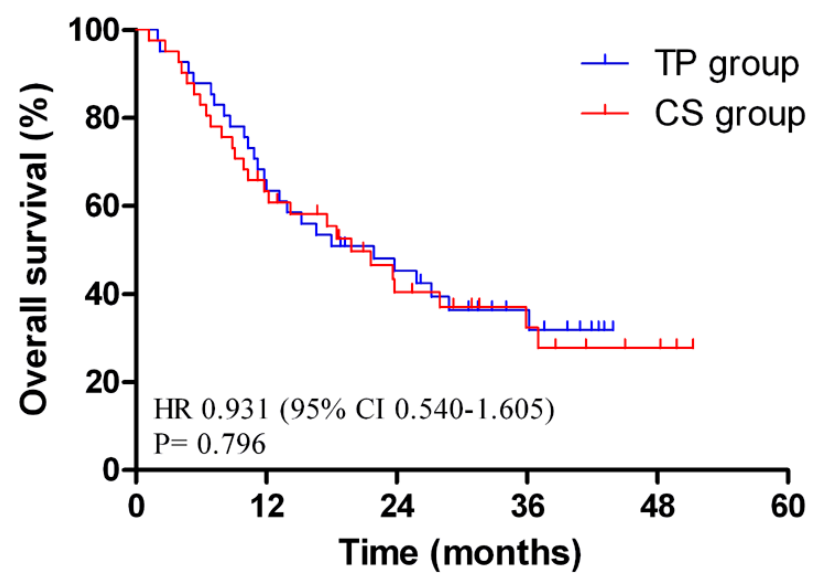

weight loss (HR 1.423, $P=0.038$ ) were significant factors associated with PFS (Supplementary Tables 2 and 3).

\section{DISCUSSION}

It has been shown previously that TP regimen combined with RT yielded favorable response rates and comparable survival outcomes $[7,8]$. However, treatmentrelated toxicities need to be taken into consideration while making the treatment plan. S-1 was an orally active fluoropyrimidine with enhanced anticancer effects and reduced gastrointestinal toxicities. It has been widely used in a variety of solid tumors, including esophageal cancer $[17,18]$. In a phase II trial performed by Cho et al., 30 locally advanced or metastatic ESCC patients were assigned to receive S-1 and CDDP at doses of $70 \mathrm{mg} /$ $\mathrm{m}^{2} /$ day for 14 days and $70 \mathrm{mg} / \mathrm{m}^{2}$ on day 1 , respectively, every 3 weeks. After CCRT, additional chemotherapy was administered up to six cycles [19]. The ORR was observed in $74.1 \%(20 / 27)$ patients. In patients at stages II-III, the median PFS and OS were 10.6 and 23.0 months, respectively, while for patients with metastatic diseases (4 patients were diagnosed with stage IVb), the median PFS and OS were 5.4 and 11.6 months, respectively. The main hematological toxicity was neutropenia (19\%, 10/54) and the major non-hematological toxicities were asthenia and vomiting, which were mostly observed for grades 1 and 2. In another prospective study, 116 LAESCC patients were allocated to receive a 30Gy RT over 3 weeks plus daily oral S-1 $\left(80 \mathrm{mg} / \mathrm{m}^{2} /\right.$ day $)$ for 2 weeks and a 24-hour CDDP infusion $\left(70 \mathrm{mg} / \mathrm{m}^{2}\right)$ on day 8 , and an identical course administered after a 2 -week break [14]. 91.4\% (106/116) of patients completed the CCRT course. ORR was achieved in $89.7 \%$ patients. The 1- and 5-year OS rates were $78.2 \%$ and $29.8 \%$, respectively, and the median PFS was 1.2 years. Grade 3 and 4 neutropenia was observed in $28.4 \%$ and $9.5 \%$ of patients, respectively. Non-hematologic toxicity was moderate. Our data were consistent with these studies. However, the incidence of total neutropenia in the TP

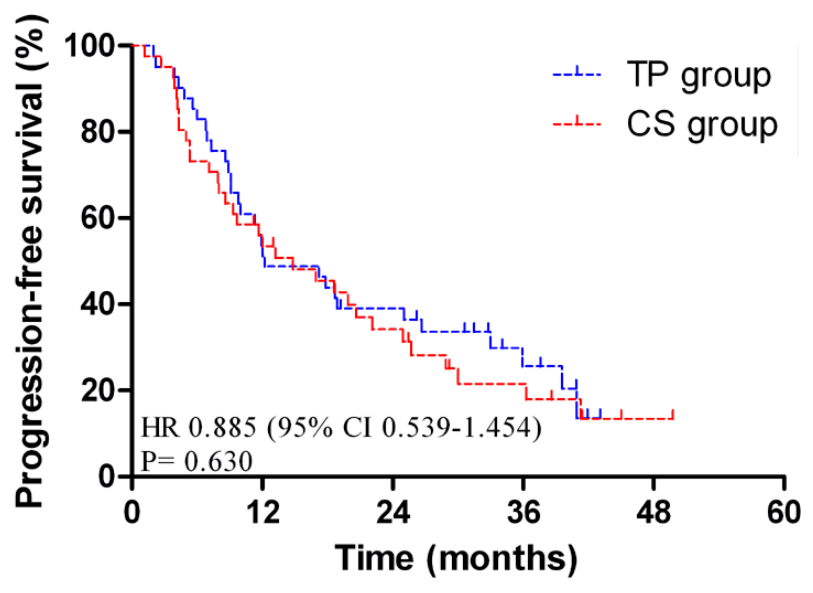

Figure 2: Comparison of overall survival (OS) and progression-free survival (PFS) in matched patients. 


\section{Table 3: Univariate analysis demonstrating factors associated with OS and PFS in matched patients}

\begin{tabular}{|c|c|c|c|c|c|}
\hline Factor & Cases (n) & OS $p$-value & HR (95\% CI) & PFS $p$-value & HR (95\% CI) \\
\hline Treatment regimen & & 0.796 & $1.074(0.624-1.851)$ & 0.630 & $1.129(0.688-1.853)$ \\
\hline $\mathrm{TP}$ & 41 & & & & \\
\hline $\mathrm{CS}$ & 41 & & & & \\
\hline Age & & 0.168 & $0.674(0.385-1.181)$ & 0.116 & $0.664(0.399-1.106)$ \\
\hline age $<58$ & 35 & & & & \\
\hline age $\geq 58$ & 47 & & & & \\
\hline Sex & & 0.428 & $1.338(0.652-2.748)$ & 0.498 & $1.265(0.641-2.499)$ \\
\hline Female & 12 & & & & \\
\hline Male & 70 & & & & \\
\hline ECOG PS & & 0.002 & $2.416(1.366-4.276)$ & 0.013 & $1.976(1.156-3.379)$ \\
\hline $0-1$ & 58 & & & & \\
\hline 2 & 24 & & & & \\
\hline T Stage & & 0.000 & $4.101(2.269-7.413)$ & 0.000 & $2.570(1.546-4.273)$ \\
\hline $\mathrm{T} 3$ & 40 & & & & \\
\hline $\mathrm{T} 4$ & 42 & & & & \\
\hline N Stage & & 0.002 & $2.387(1.365-4.172)$ & 0.002 & $2.304(1.369-3.876)$ \\
\hline N0 & 46 & & & & \\
\hline N1 & 36 & & & & \\
\hline M Stage & & 0.010 & $2.129(1.195-3.792)$ & 0.058 & $1.711(0.982-2.981)$ \\
\hline M0 & 58 & & & & \\
\hline M1 & 24 & & & & \\
\hline Clinical Stage & & 0.000 & $1.892(1.321-2.708)$ & 0.009 & $1.537(1.111-2.125)$ \\
\hline II & 25 & & & & \\
\hline III & 33 & & & & \\
\hline $\mathrm{IV}_{\mathrm{a}}$ & 24 & & & & \\
\hline Tumor Location & & 0.173 & $1.305(0.890-1.913)$ & 0.082 & $1.364(0.061-1.937)$ \\
\hline Upper-third & 38 & & & & \\
\hline Middle-third & 30 & & & & \\
\hline Lower-third & 14 & & & & \\
\hline Differentiation & & 0.255 & $0.806(0.556-1.169)$ & 0.327 & $0.842(0.597-1.187)$ \\
\hline Well & 19 & & & & \\
\hline Fairly & 37 & & & & \\
\hline Poorly & 26 & & & & \\
\hline Tumor Length (cm) & & 0.252 & $1.396(0.789-2.470)$ & 0.082 & $1611(0.941-2.758)$ \\
\hline$<5$ & 32 & & & & \\
\hline$\geq 5$ & 50 & & & & \\
\hline Weight Loss & & 0.479 & $1.233(0.690-2.203)$ & 0.683 & $1.119(0.653-1.919)$ \\
\hline$\leq 10 \%$ & 57 & & & & \\
\hline$>10 \%$ & 25 & & & & \\
\hline
\end{tabular}


Non-CR

41

Abbreviations: $n$ : number of patients; OS: overall survival; PFS: progression-free survival; HR: hazard ratio; CI: confidence interval, CR: complete response.

\section{Table 4: Multivariate analysis of prognostic factors for matched patients}

\begin{tabular}{llcc}
\hline Endpoint & Factor & $\boldsymbol{p}$-value & HR (95\% CI) \\
\hline $\boldsymbol{O S}$ & T Stage & 0.009 & $3.420(1.365-8.569)$ \\
& N Stage & 0.027 & $2.270(1.098-4.692)$ \\
M Stage & 0.072 & $3.571(0.891-14.307)$ \\
Clinical Stage & 0.229 & $0.498(0.160-1.551)$ \\
& ECOG PS & 0.128 & $1.596(0.875-2.912)$ \\
& Clinical Response & 0.002 & $2.860(1.488-5.497)$ \\
$\boldsymbol{P F S}$ & ECOG PS & 0.239 & $1.413(0.795-2.511)$ \\
& T Stage & 0.042 & $2.083(1.026-4.229)$ \\
& N Stage & 0.014 & $2.295(1.184-4.450)$ \\
& Clinical Stage & 0.718 & $0.904(0.522-1.566)$ \\
& Clinical Response & 0.012 & $2.061(1.175-3.613)$ \\
\hline
\end{tabular}

Abbreviations: OS: overall survival; PFS: progression-free survival; HR: hazard ratio; CI: confidence interval.

group was not significantly higher during maintenance chemotherapy in our study, one probable reason to explain this phenomenon was that chemotherapy combined with radiation therapy had superposition effect during CCRT, which would increase the incidence of toxic reactions [19].

To date, although there were no direct comparisons between TP and CS for esophageal cancer, a similar comparison of PTX versus S-1 was observed in non-small cell lung cancer (NSCLC) patients. A multicenter phase 3 study randomizing stage IIIb-IV NSCLC patients to PTX/carboplatin versus S-1/carboplatin chemotherapy was performed [21]. The median OS was 15.2 versus 13.3 months in the S-1/carboplatin and PTX/carboplatin arm, with 1-year OS rates of $57.3 \%$ and $55.5 \%$, respectively. The rates of grade $3 / 4$ leukopenia or neutropenia, febrile neutropenia, alopecia, and neuropathy were more frequently observed in the PTX/carboplatin group. It was concluded that S-1 with carboplatin was not inferior in terms of OS compared with carboplatin and PTX in patients with advanced NSCLC and was thus a valid treatment option.

In the present study, we confirmed that the $\mathrm{T}$ stage was a strong prognostic factor for both OS and PFS before and after PSM analysis, which was consistent with previous reports in the literature [22, 23]. In addition, patients who were evaluated as a complete response were also confirmed to have longer survival times compared with non-CR patients [24].
This study has some limitations. One limitation of the current study is the retrospective nature of the study design. Although propensity score matching was performed to reduce potential influence, some unmeasured factors might have effects on the final results. Furthermore, another limitation of current study was the relatively small sample size and low-level of power performed in a single center.

In conclusion, both the TP and CS regimens yielded encouraging survival outcomes and manageable side effects. However, the CS regimen could significantly improve treatment compliance, reduce hematological toxicities and lengths of hospital stay. On the basis of the findings of this propensity score matching analysis, future prospective trials in large cohorts are highly needed to confirm the findings (i.e., NCT01704690).

\section{MATERIALS AND METHODS}

\section{Patient work-up}

This retrospective study was approved by the ethical committee of Zhejiang Provincial People's Hospital and the written informed consent was obtained from all patients. Between January 2009 and December 2013, 376 consecutive patients with newly diagnosed esophageal cancer were reviewed at our cancer center. Inclusion in our review included the following criteria: i) a histological 
diagnosis of esophageal squamous cell carcinoma; ii) disease stages of II to IVa according to the 2002 (version 6.0) American Joint Committee on Cancer staging system; iii). unresectable diseases or refusal of surgery after being discussed by the multidisciplinary treatment team; iv) an ECOG PS of at least 2; v) no evidence of severe organ dysfunction; and vi) no prior chest radiation or chemotherapy received.

\section{Treatment}

\section{Radiotherapy}

Radiotherapy was delivered with 6-10 Mv X-ray accelerators using the three-dimensional conformal technique (3D-CRT). The gross tumor volume (GTV) included the primary tumor and any enlarged lymph nodes. For regional lymph nodes, the supraclavicular, upper mediastinal, and subcarinal lymph nodes were irradiated for tumors in the proximal esophagus. The mediastinal and perigastric lymph nodes were included for tumors of the middle or lower esophagus, in which the celiac lymph nodes were added for lower-segment cancers. The clinical target volume (CTV) was defined as the GTV plus a 4-5 cm margin in the superior and inferior directions, $1 \mathrm{~cm}$ in the left and right directions and $0.8-1 \mathrm{~cm}$ in the anterior and posterior directions. The planning target volume (PTV) was defined as the CTV plus a 5-10 mm margin around the CTV. The preplanned total dose was $60.0 \mathrm{~Gy}$, which was administered in 30 fractions of 2.0 Gy oncedaily fractions for 5 days per week.

\section{Chemotherapy}

Two cycles of concurrent chemotherapy were delivered with RT. CDDP $\left(30 \mathrm{mg} / \mathrm{m}^{2}\right)$ was administered intravenously on days 1-3 and days 29-31 for all enrolled patients [25]. The chemotherapy regimen of TP group patients was as follows: PTX $135 \mathrm{mg} / \mathrm{m}^{2}, i . v$. was administered for 3 hours on day 1 and day 29 with standard premedications. The chemotherapy regimen of CS group patients: S-1 was administered orally twice daily for 2 weeks at a dose of $70 \mathrm{mg} / \mathrm{m}^{2} /$ day. Patients who showed a response greater than that of stable disease underwent additional four cycles of chemotherapy.

\section{Dose modification}

Dose modifications were considered on a weekly basis during CCRT. Chemotherapy was delayed for acute toxicities until recovery to grades $\leq 2$ and/or the dose was reduced for grade 3 or higher hematological toxicity. PTX was reduced to $80 \%$ in the second course if any of the following phenomena occurred: grade 3 leukocytopenia with fever or grade 4 leukocytopenia. The granulocyte colony-stimulating factor (G-CSF) was used to treat the occurrence of febrile neutropenia. If the creatinine clearance decreased to less than $50 \mathrm{~mL} / \mathrm{min}$, then the dose of CDDP was reduced to $75 \%$. S-1 was suspended due to excessive toxicity or patient request. In cases of severe toxicity related with $\mathrm{S}-1$, interruptions were allowed up to 14 days. If the side effects did not return to grades $\leq$ 2 , then a dose reduction by $60 \mathrm{mg} / \mathrm{m}^{2} /$ day was allowed, but no more than one dose reduction was permitted. Irradiation was interrupted for grade $\geq 3$ esophagitis, grade 3 leukocytopenia with fever, or grade 4 leukocytopenia. Radiotherapy was restarted when toxicities recovered to grades $\leq 2$.

\section{Treatment assessment and follow-up}

Treatment related toxicity was evaluated according to the common toxicity criteria for adverse events version 3.0 (CTCAE v3.0). We evaluated the clinical response of patients using the Response Evaluation Criteria in Solid Tumors (RECIST) 3-4 weeks after the completion of CCRT. Follow-up was regularly performed at 3 months during the first year, then every 6 months for 2 additional years, and then on a yearly basis. Treatment failure was defined as any sign of recurrent disease, which could be locoregional, distant, or both. We assessed failure models on post-treatment esophagogram, endoscopy, CT, or PET/ CT (if available) scans and compared these data with the original CT-based treatment plans.

\section{Statistical analysis}

To reduce bias of potential confounders between the two groups, propensity score matching was performed to generate two treatment cohorts with a balanced distribution of baseline characteristics. The following variables were included in a logistic regression model, regardless of their individual statistical significance: gender; ECOG PS; T stage; $M$ stage; clinical stage; tumor length; and weight loss over the nearly 6 month-study period. The potential predictors that were not statistically significant were removed (Supplementary Table 1), and propensity scores were calculated from the logistic regression refit to the reduced variable group. Only patients matched with propensity scores were included in the subsequent analyses.

Covariate balances between the two sets were examined by $t$ test (continuous variable), Mann-Whitney $\mathrm{u}$ test (abnormal distribution variable), $\chi^{2}$ test or Fisher's exact test (categorical variable) as appropriate. OS was determined as the time between the first day of treatment and the last follow-up or the date of death. PFS was defined as the internal between the date of treatment initiation and the date of documented failure or the date of the last follow-up for those remaining. Survival curves were determined using the Kaplan-Meier method. Predictive factors of survival were analyzed using univariate analysis and further evaluated in the multivariate Cox regression model to estimate the hazard ratio (HR) with a 95\% confidence interval (CI). All statistical calculations were 
performed using STATA 12.0 (Stata Corporation, College Station, TX, USA) and Statistical Package for the Social Sciences (SPSS 20.0, Inc., Chicago; for Windows). A $P$ value $<0.05$ was considered statistically significant.

\section{CONFLICTS OF INTEREST}

None.

\section{REFERENCES}

1. Siegel R, Ma J, Zou Z, Jemal A. Cancer statistics, 2014. CA Cancer J Clin. 2014; 64:9-29.

2. Bray F, Jemal A, Grey N, Ferlay J, Forman D. Global cancer transitions according to the Human Development Index (2008-2030): a population-based study. The Lancet Oncology. 2012; 13:790-801.

3. Chen W, Zheng R, Baade PD, Zhang S, Zeng H, Bray F, Jemal A, Yu XQ, He J. Cancer statistics in China, 2015. CA Cancer J Clin. 2016.

4. Cooper JS, Guo MD, Herskovic A, Macdonald JS, Martenson JA Jr, Al-Sarraf M, Byhardt R, Russell AH, Beitler JJ, Spencer S, Asbell SO, Graham MV, Leichman LL. Chemoradiotherapy of locally advanced esophageal cancer: long-term follow-up of a prospective randomized trial (RTOG 85-01). Radiation Therapy Oncology Group. Journal of the american medical association. 1999; 281:1623-1627.

5. Orditura M, Galizia G, Napolitano V, Martinelli E, Pacelli R, Lieto E, Aurilio G, Vecchione L, Morgillo F, Catalano G, Ciardiello F, Del Genio A, Di Martino N, et al. Weekly chemotherapy with cisplatin and paclitaxel and concurrent radiation therapy as preoperative treatment in locally advanced esophageal cancer: a phase II study. Cancer Investigation. 2010; 28:820-827.

6. Li QQ, Liu MZ, Hu YH, Liu H, He ZY, Lin HX. Definitive concomitant chemoradiotherapy with docetaxel and cisplatin in squamous esophageal carcinoma. Diseases of the Esophagus. 2010; 23:253-259.

7. Ajani JA, Winter K, Komaki R, Kelsen DP, Minsky BD, Liao Z, Bradley J, Fromm M, Hornback D, Willett CG. Phase II randomized trial of two nonoperative regimens of induction chemotherapy followed by chemoradiation in patients with localized carcinoma of the esophagus: RTOG 0113. Journal of Clinical Oncology. 2008; 26:4551-4556.

8. Tang HR, Ma HF, An SM, Badakhshi H, Deng JY, Zhang JH, Chen Y, Zhang Z, Guo XM, Jiang GL, Zhao KL. A phase II study of concurrent chemoradiotherapy with paclitaxel and cisplatin for inoperable esophageal squamous cell carcinoma. American Journal of Clinical Oncology. 2016; 39:350-354.

9. Marupudi NI, Han JE, Li KW, Renard VM, Tyler BM, Brem H. Paclitaxel: a review of adverse toxicities and novel delivery strategies. Expert Opinion on Drug Safety. 2007; 6:609-621.
10. Fukushima M, Sakamoto K, Sakata M, Nakagawa F, Saito H, Sakata Y. Gimeracil, a component of S-1, may enhance the antitumor activity of X-ray irradiation in human cancer xenograft models in vivo. Oncology Reports. 2010; 24:1307-1313.

11. Harada K, Kawaguchi S, Supriatno, Kawashima Y, Yoshida H, Sato M. S-1, an oral fluoropyrimidine anticancer agent, enhanced radiosensitivity in a human oral cancer cell line in vivo and in vitro: involvement possibility of inhibition of survival signal, Akt/PKB. Cancer Letters. $2005 ; 226: 161-168$.

12. Li YH, Qiu MZ, Xu JM, Sun GP, Lu HS, Liu YP, Zhong MZ, Zhang HL, Yu SY, Li W, Hu XH, Wang JJ, Cheng Y, et al. S-1 plus cisplatin versus fluorouracil plus cisplatin in advanced gastric or gastro-esophageal junction adenocarcinoma patients: a pilot study. Oncotarget. 2015; 6:35107-35115. doi: 10.18632/oncotarget.5959.

13. Chang H, Shin SK, Cho BC, Lee CG, Kim CB, Kim DJ, Lee JG, Hur J, Lee CY, Bae MK, Kim HR, Lee SK, Park JC, et al. A prospective phase II trial of S-1 and cisplatin-based chemoradiotherapy for locoregionally advanced esophageal cancer. Cancer Chemotherapy and Pharmacology. 2014; 73:665-671.

14. Iwase H, Shimada M, Tsuzuki T, Hirashima N, Okeya M, Hibino Y, Ryuge N, Yokoi M, Kida Y, Kuno T, Tanaka Y, Kato B, Esaki M, et al. Concurrent chemoradiotherapy with a novel fluoropyrimidine, S-1, and cisplatin for locally advanced esophageal cancer: long-term results of a phase II trial. Oncology. 2013; 84:342-349.

15. Tahara M, Fuse N, Mizusawa J, Sato A, Nihei K, Kanato K, Kato K, Yamazaki K, Muro K, Takaishi H, Boku N, Ohtsu A. Phase I/II trial of chemoradiotherapy with concurrent S-1 and cisplatin for clinical stage II/III esophageal carcinoma (JCOG 0604). Cancer Science. 2015; 106:1414-1420.

16. Tang HR, Ma HF, An SM, Badakhshi H, Deng JY, Zhang JH, Chen Y, Zhang Z, Guo XM, Jiang GL, Zhao KL. A phase II study of concurrent chemoradiotherapy with paclitaxel and cisplatin for inoperable esophageal squamous cell carcinoma. American Journal of Clinical Oncology. 2014.

17. van Groeningen CJ, Peters GJ, Schornagel JH, Gall H, Noordhuis P, de Vries MJ, Turner SL, Swart MS, Pinedo HM, Hanauske AR, Giaccone G. Phase I clinical and pharmacokinetic study of oral S-1 in patients with advanced solid tumors. Journal of Clinical Oncology. 2000; 18:2772-2779.

18. Yamada Y, Hamaguchi T, Goto M, Muro K, Matsumura Y, Shimada Y, Shirao K, Nagayama S. Plasma concentrations of 5-fluorouracil and F-beta-alanine following oral administration of S-1, a dihydropyrimidine dehydrogenase inhibitory fluoropyrimidine, as compared with protracted venous infusion of 5-fluorouracil. British Journal of Cancer. 2003; 89:816-820. 
19. Cho SH, Shim HJ, Lee SR, Ahn JS, Yang DH, Kim YK, Nam TK, Lee JJ, Kim HJ, Chung IJ. Concurrent chemoradiotherapy with S-1 and cisplatin in advanced esophageal cancer. Diseases of the Esophagus. 2008; 21:697-703.

20. Ji Y, Qiu G, Sheng L, Sun X, Zheng Y, Chen M, Du X. A phase I dose escalation study of S-1 with concurrent radiotherapy in elderly patients with esophageal cancer. Journal of Thoracic Disease. 2016; 8:451-458.

21. Okamoto I, Yoshioka H, Morita S, Ando M, Takeda K, Seto T, Yamamoto N, Saka H, Asami K, Hirashima T, Kudoh S, Satouchi M, Ikeda N, et al. Phase III trial comparing oral S-1 plus carboplatin with paclitaxel plus carboplatin in chemotherapy-naive patients with advanced non-small-cell lung cancer: results of a west Japan oncology group study. Journal of Clinical Oncology. 2010; 28: 5240-5246.

22. Liu M, Chen Y, Fu X, Zhao K, Jiang GL. Proposed revision of CT-based cervical and thoracic lymph node levels for esophageal cancer in UICC 7th version. Radiotherapy and Oncology. 2014; 113:175-181.
23. Nomura M, Shitara K, Kodaira T, Hatooka S, Mizota A, Kondoh C, Yokota T, Takahari D, Ura T, Muro K. Prognostic impact of the 6th and 7th American Joint Committee on Cancer TNM staging systems on esophageal cancer patients treated with chemoradiotherapy. International Journal of Radiation Oncology, Biology, Physics. 2012; 82:946-952.

24. Di Fiore F, Blondin V, Hitzel A, Edet-Sanson A, Benyoucef A, Huet E, Vera P, Michel P. 18F-fluorodeoxyglucose positron emission tomography after definitive chemoradiotherapy in patients with oesophageal carcinoma. Digestive and Liver Disease. 2012; 44:875-879.

25. Song $T$, Zhang $X$, Fang $M$, Wu $S$. Concurrent chemoradiotherapy using paclitaxel plus cisplatin in the treatment of elderly patients with esophageal cancer. Oncotargets and Therapy. 2015; 8:3087-3094. 